$678.077: 677$

\title{
Elastic Index of the Work-Done of FRP*
}

\author{
By Tsuneo Hirai**, Shoichi Fujiwara***, and Shigehisa Otsuki****
}

The elasto-plastic behavior of fiber-glass reinforced plastics (FRP) appears to be caused by the cracks progressing along the interface between different constituents and propagating across the matrix. This paper reports an investigation on the relationship between the value of strain applied in the FRP specimen and the state of micro-fracture by considering the value of elastic index of work-done (E.I.W.).

For the plastics reinforced by the glass fabrics which has systematic structure, the E.I.W. decreased rapidly at the knee point. After the strain passed the knee, it recovered again, and even a small reduction occured in a certain region. The large reduction of the F.I.W. at the knee is due to the cracks inside of woof strands, and the later small reduction is due to the cracks inside of warp strands.

Specially the transition of E.I.W. by step-wise increasing of strain shows the progress and propagation of cracks in the corresponding parts of FRP.

\section{Introduction}

The analysis and design of the glass-fiber reinforced plastics (FRP) are more difficult than those of ordinary materials because their elasto-plastic behavior are the complex functions of the material properties, the geometric arrangement of the reinforced material and the interface between different constitutents. To make clear the mechanical properties of FRP, some investigations were carried out theoretically and experimentally by the microscopic observation and the theoretical analysis of it as an anisotropic semi-homogenious material ${ }^{(1) \sim(4)}$.

Before the mechanical properties are considered in detail, it should be recognized that the behavior of FRP depends on its micro-structure which develops due to the progress and propagation of cracks in the interface of composite materials by the corresponding strain. To measure the transition of elastic index of the work-done (E.I.W.) corresponding to the strain increment, it is helpful to settle the above problem.

Here, we have a nomenclature named "elastic index of the work-done" (E.I.W.) which is defined as a ratio of the whole work-done and the elastic work-done for the various stages of strain of FRP. Then from the tendency in the change of E.I.W. with strain, we considered the behaviors of stressed reinforcement and matrix, especially the plastic behavior caused by the cracks growing in FRP. We examined

* Received 3rd October, 1969.

** Professor, Faculty of Technology Doshisha University.

*** Engineer, Industrial Research Institute Osaka Pref., Sakai.

**** Chief of Laboratory, Kansai Slate Co., Ltd. the E.I.W. of the plastics reinforced by various kinds of glass fabrics, and studied the dynamic behaviors of FRP. For the reinforcement, we used the roving plain fabrics mainly because it was easier to observe the behavior in them than in the other fabrics.

\section{Material of specimen}

The resin used for the specimen of FRP is an unsaturated polyester (Epolac N-340). And the specifications of glass woven fabrics used as the reinforcement are shown in Table 1.

For preparing the specimen, a metal die was used. At first, the resin and the glass fabrics were put in to the metal die and after that, the metal die was put into a vacuum device for clearing away the foams within the resin. After that, the forming pressure was given to the metal die. By using such processes $^{(5)}$, we endeavored to get homogeneous laminates. The thickness of laminates was about $6 \mathrm{~mm}$. The after-cure was performed for 24 hours at $95^{\circ} \mathrm{C}$ on all laminates. This condition was chosen as the best one from the former results ${ }^{(6)}$. The kind of specimens and each rate of the volumetric glass content $\beta$ of them are shown respectively in Table 2 .

In the calculation of $\beta$, the following equation ${ }^{(7)}$ was used.

$$
\beta=\frac{n \omega}{t_{l} \rho_{g}}
$$

where $N$ : number of sheets of glass woven fabrics in the laminate.

$t_{l}$ : thickness of the laminate $\mathrm{cm}$

$\rho_{g}$ : density of the glass fiber $\mathrm{g} / \mathrm{cm}^{3}$

$w$ : weight of glass woven fabrics per unit 
area $\mathrm{g} / \mathrm{cm}^{2}$

The difference between the calculated value $\beta$ by using the above equation and the observed value is less than $1 \%$, so the above equation does not cause any inconvenience in practical use.

For the relation with mechanical properties of FRP, the volumetric ratio of glass fiber is more suitable

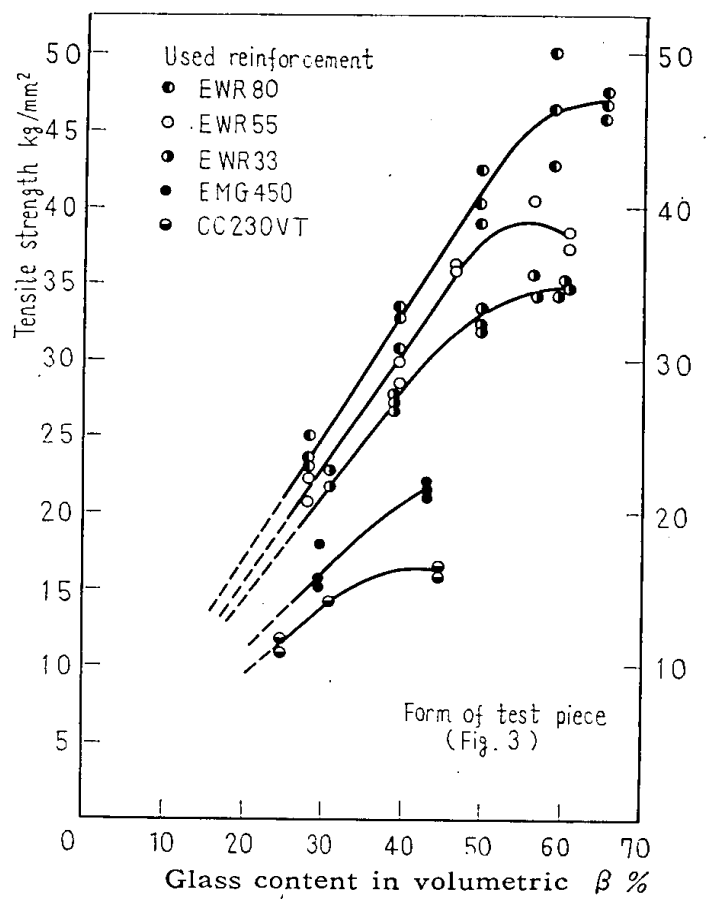

Fig. I Relation of tensile strength vs. glass content by volume $\beta$ about used FRP

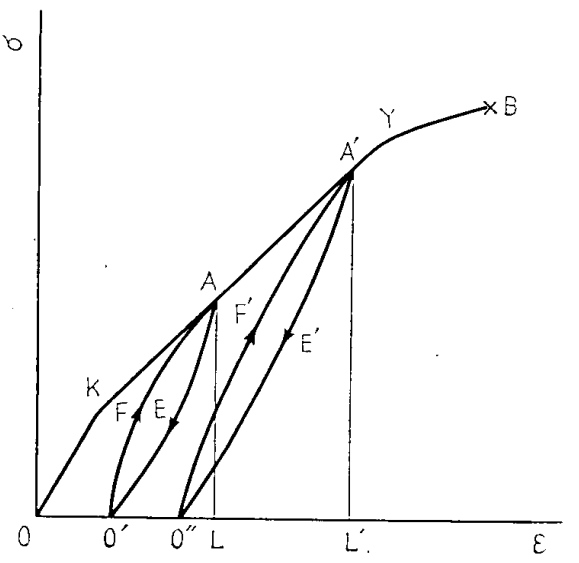

Fig. $2 \quad \sigma-\varepsilon$ diagram than its weight ratio. For that reason, we adopted the volumetric ratio of glass fiber. The relation. between $\beta$ and the tensile strength of the specimen of FRP is shown in Fig. 1.

\section{Elastic index of the work-done}

When the FRP is subjected to a tensile load, it. is well known that the stress-strain diagram follows. the route of OKAYB in Fig. 2 and breaks at the point B. In the first stage, from 0 to the point $K$ (which is called "knee"), it is almost elastic. In the stage of KY, a transformation occurs in the construction. within the material, and OKY line becomes approximately as a broken line. In the stage from $\mathrm{Y}$, the diagram bas a curvature and reachs the point $B$.

Here, if the load is released at the point $A$ between. $\mathrm{K}$ and $\mathrm{Y}$, the unloading curve goes to $\mathrm{O}^{\prime}$ through $\mathrm{E}$. And when the material is subjected to the load again, the diagram returns to A through F. Further, if the stress acts alternately at point $\mathrm{A}$, the diagram makes a loop of O'FAEO'. This loop moves to the right a. little, and is stabilized after a few cycles of the alternating stress ${ }^{(8)}$. And it seems that this stable state continues just before the fatigue rupture happens. In this case, the E.I.W. on the strain OL is expressed: as follows:

$$
\begin{aligned}
\frac{W_{e}}{W_{i}} & =\frac{\text { area } \mathrm{AEO} \mathrm{LA}^{\prime}}{\text { area } \mathrm{OKALO}} \\
& =\frac{\text { work-done recovered by elasticity }}{\text { work-done given to virgin material }} \\
\frac{W_{e}}{W} & =\frac{\text { area } \mathrm{AEO} \mathrm{LA}}{\text { area } \mathrm{O}^{\prime} \mathrm{FALO}} \\
& =\frac{\text { work-done recovered by elasticity }}{\text { work-done alternately given on each strain }}
\end{aligned}
$$

Furthermore, as regards the point $\mathrm{A}^{\prime}$, also the same tendency exists. In this report, to make clear the

\begin{tabular}{|c|c|c|c|c|c|}
\hline \multirow{2}{*}{$\begin{array}{c}\text { Name of } \\
\text { fabrics }\end{array}$} & \multirow[b]{2}{*}{ Woven structure } & \multirow{2}{*}{ Thickness mm } & \multicolumn{2}{|c|}{ Density } & \multirow{2}{*}{$\begin{array}{c}\text { Standard } \\
\text { weight } g / \mathrm{m}^{2}\end{array}$} \\
\hline & & & $\begin{array}{l}\text { No. of } \\
\text { warp/in }\end{array}$ & $\begin{array}{l}\text { No. of } \\
\text { woof/in }\end{array}$ & \\
\hline EWR33 & Roving plain woven & 0.37 & 14.5 & 12.5 & 344.8 \\
\hline EWR55 & " & 0.59 & 6.5 & 6.0 & 590.3 \\
\hline EWR80 & $" \prime$ & 0.79 & 4.5 & 4.5 & 809.0 \\
\hline CC230VT & Plain woven & 0.25 & 19 & 19 & 220 \\
\hline EMG 450 & Non-woven & & & & 450 \\
\hline
\end{tabular}

Table 2 Kinds of work-piece

\begin{tabular}{l|r|c}
\hline $\begin{array}{c}\text { Kind of } \\
\text { reinforcement }\end{array}$ & Number of plies & $\begin{array}{c}\text { Glass content in } \\
\text { volumetric per- } \\
\text { centage } \beta \% \\
\text { (approximately) }\end{array}$ \\
\hline EWR33 & $13,17,22,26,30$ & $30,39,49,56,58$ \\
EWR55 & $7,10,12,15,17$ & $28,40,49,56,61$ \\
EWR80 & $5,7,9,11,13$ & $28,39,50,59,64$ \\
CC230VT & 19,38 & 25, \\
EMG450 & \multicolumn{2}{|c|}{25} \\
\hline
\end{tabular}

Table 1 Kinds and specification of glass fabrics 
dynamic behaviors of FRP, the plastic behavior of the test piece caused by the strain energy with each small increment of strain was also investigated. And the following value on each strain as calculated.

$$
\begin{aligned}
\frac{W_{t}}{W_{t}} & =\frac{\text { area } \mathrm{A}^{\prime} \mathrm{E}^{\prime} \mathrm{O}^{\prime \prime} \mathrm{L}^{\prime} \mathrm{A}^{\prime}}{\text { area } \mathrm{O}^{\prime} \mathrm{FAA} \mathrm{A}^{\prime} \mathrm{L}^{\prime} \mathrm{O}^{\prime}} \\
& =\frac{\text { work-done recovered by elasticity }}{\text { work-done given from strain } \mathrm{L} \text { to } \mathrm{L}^{\prime}}
\end{aligned}
$$

The secant modulus on $\widehat{\mathrm{O}^{\prime} \mathrm{A}}$ or $\widehat{\mathrm{O}^{\prime \prime} \mathrm{A}}$ ' has a tendency to decrease as the strain increases. Here, it is presumed that a small amount of delayed recovery exists in the test, but it is neglected in this report.

The form of the test piece used for the measurement of the E.I.W. was a strip-shape as shown in Fig. 3. According to the inference drawn from another expcrimental results by the present authors( ${ }^{(4)}$, the strip-shaped test piece is more faithful in the detection of the tensile behavior of FRP than the conventional one. For that reason, the strip-shaped test pieces were used. And they were picked up so that the loading might be performed in the direction of the warp strand.

For the measurement, an universal testing machine of strain control type was used. In the detection of the load, a strain gauge transducer was used in the chuck part, and in the detection of strain, an extensometer of differential transformer type of $20 \mathrm{~mm}$ gauge length was used. Then stress-strain diagrams were recorded automatically.

An example of the stress-strain diagram is shown in Fig. 4. This is a result about the test piece with the reinforcement EWR55 in which the loading cycles have been repeated from $0.1 \%$ strain to $1.5 \%$ strain at appropriate intervals. Hence, $W_{\varepsilon}, W_{i}$ and $W_{t}$ are measured by weighing the piece of each corresponding figure cut out from the copied sheets of the diagram.

$W_{\imath} / W_{i}, W_{\imath} / W_{t}, E_{s}$ and $W_{h}$ are entered in the E.I.W.-strain diagram of each test piece in Fig. 5 and the succeeding figures. $W_{e} / W_{i}$ shows the synthetic variation of the E.I.W., and $W_{e} / W_{t}$ shows the variation of the E.I.W. at each increment of strain. By this $W_{t} / W_{t}$, the plastic behaviors on the test pieces may be found easily. $E_{s}$ shows the variation of the strain amount. And, by the measurement of $W_{h}$, the variation of $W_{e} / W_{t}$ and $E_{s}$ can be confirmed.

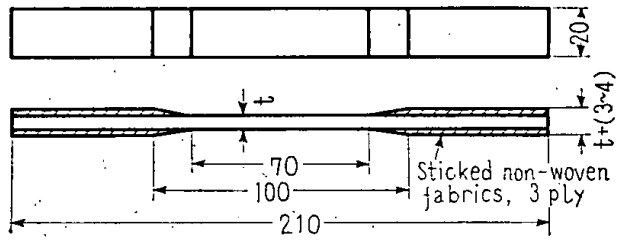

Fig. 3 Form of test piece
4. Elastic index of the work-done and its change in the plastics reinforced by the roving plain fabrics (R-FRP)

\subsection{Relationship with the behavior of the} knee

The results of the experiment on E.I.W. about R-FRP reinforced individually by three kinds of roving plain fabrics are shown in Figs. 5 to 7 . From the curves of $W_{e} / W_{i}$ and $W_{e} / W_{t}$ in these figure, we

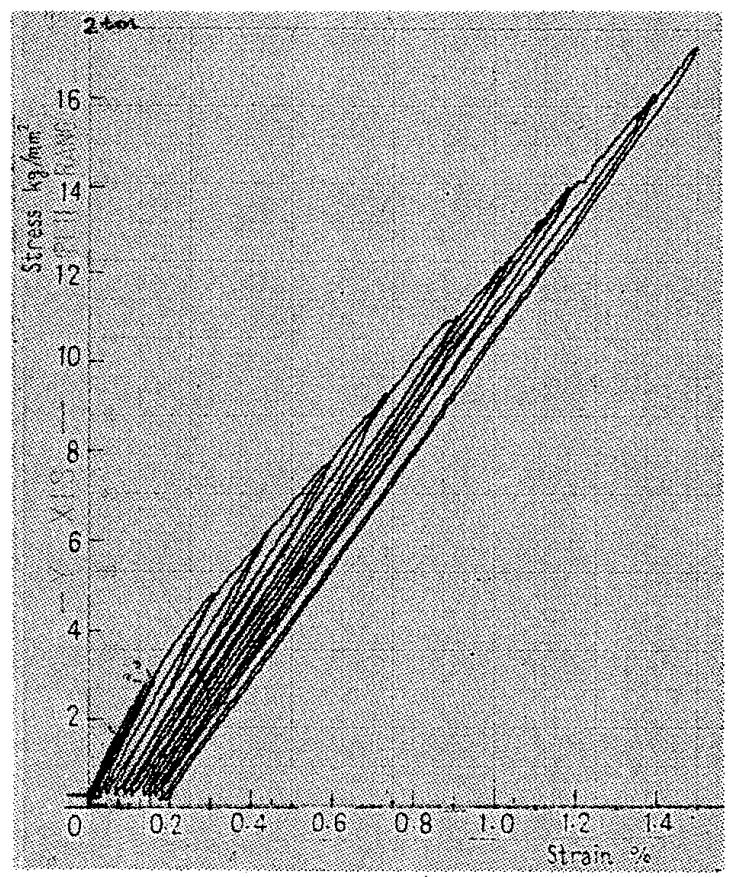

Reinforcement EWR55, $\beta=28 \%, \dot{\varepsilon}=0.20 \mathrm{~min}^{-1}$

Fig. 4 A sample of stress-strain diagrams

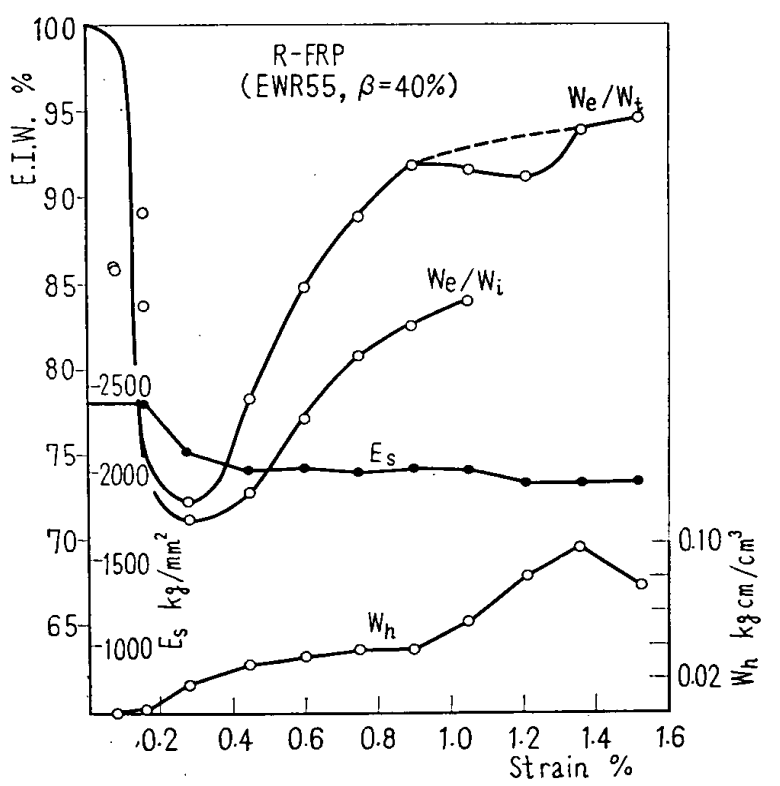

R.FRP (EWR 55), $\beta=40 \%, \dot{\varepsilon}=0.20 \mathrm{~min}$ Fig. 5 E.I.W. (No. 1) 
can find that each E.I.W. of these FRP is changed in a special process related to the strain rate. This variation may be caused by the woven structure of the reinforcement.

Until the strain value reaches $0.1 \sim 0.15 \%$, their E.I.W. show the behavior as an elastic material which is composed semi-homogeneously by both glassfiber and resin. But with respect to the strain over this value, the $W_{e} / W_{t}$ curve goes down rapidly and shows a minimum value at $0.2 \sim 0.4 \%$ strain range.

The cracks grow inside of woof strands for a very small strain, and increase rapidly with an increasing strain as mentioned later on. So, the flexural rigidity of the woof strand decreases, then the deforemations of the woof and warp strands become easy together. As the result, the woof strand shows a meandering movement and the warp strand shows a linear movement. This region where the stress value is about $4 \mathrm{~kg} / \mathrm{mm}^{2}$ and the secant modulus decreases rapidly corresponds to that about $\mathrm{K}$ in Fig. 2. This point $\mathrm{K}$ is called the knee conventionally.

The stress for one ply in the FRP is illustrated in Fig. 8, and the woof strand receives both tensile and flexural loads as shown in the figure. In this case, the woof strand is not the fiber placed in the direction of load, and the stress concentration may occur at a face of each mono-filament according to mutual positions and space of the adjoining filament. Therefore, the cracks grow for a small strain value.

Figure 9 shows the first stage of the growth of cracks, which consists of some debondings caused at a face of mono-filament and the cracks which have grown in joining them together.

The strain value which shows the minimum value of E.I.W. and that at the knee point obtained from

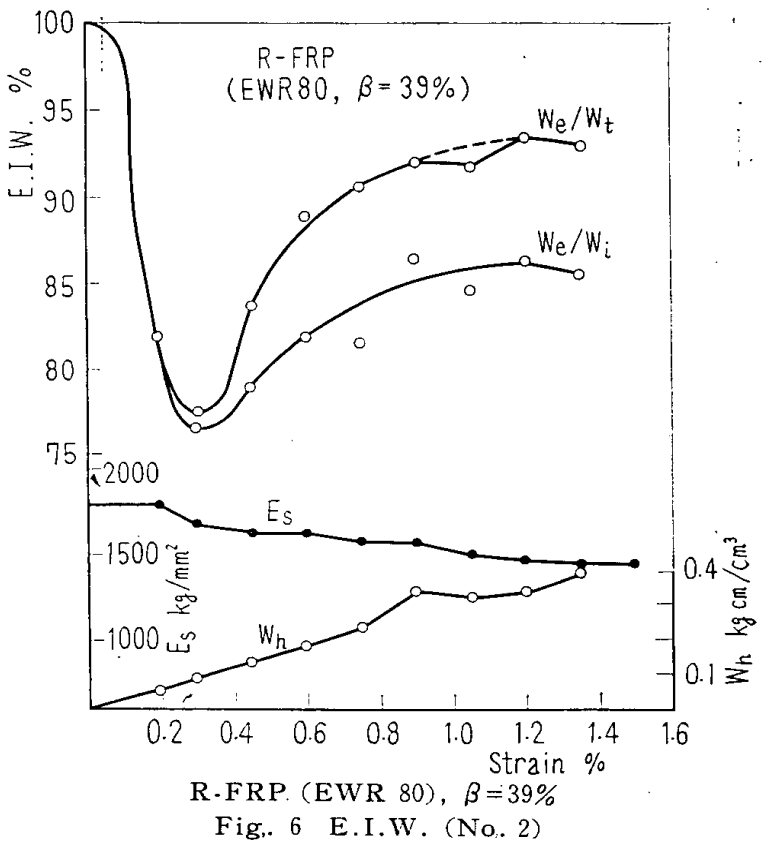

the stress-strain diagrams are plotted in relation to the value of $\beta$ of the specimen in Fig. 10 . Both curves agree approximately, and the relationship between the decrease of $W_{\theta} / W_{t}$ and knee is clearly observed. That is, it can be said that the decrease in the value of E.I.W. and the appearance of the

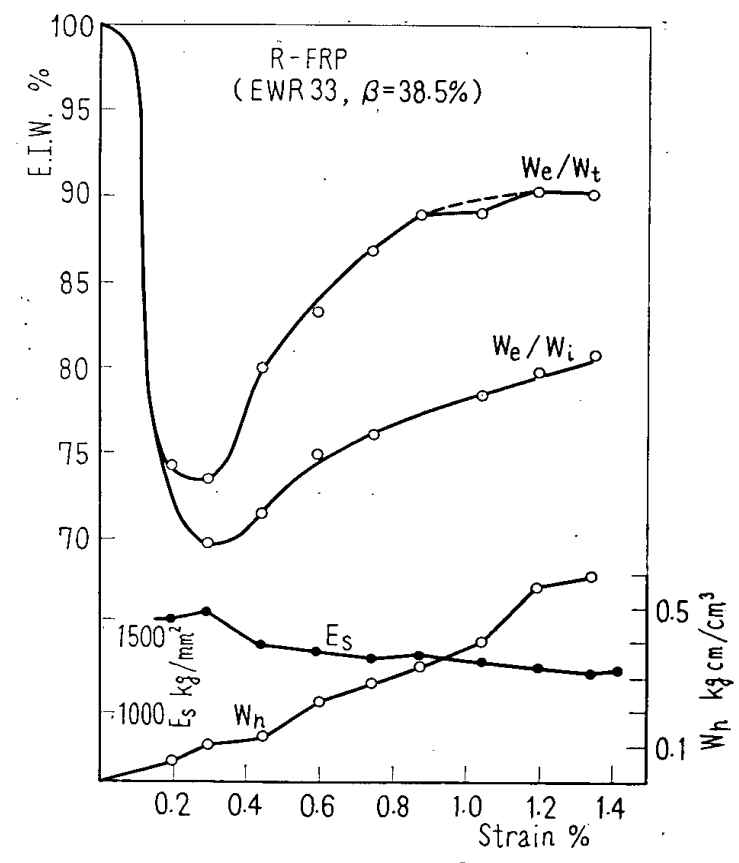

R-FRP (EWR 33), $\beta=38.5 \%$

Fig. 7 E.I.W. (No. 3)

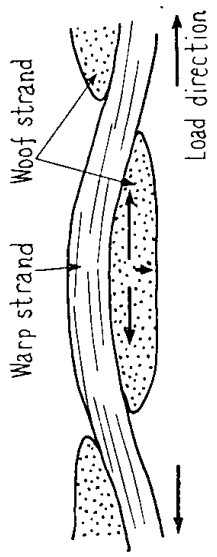

Fig. 8 Model for one ply of fabrics

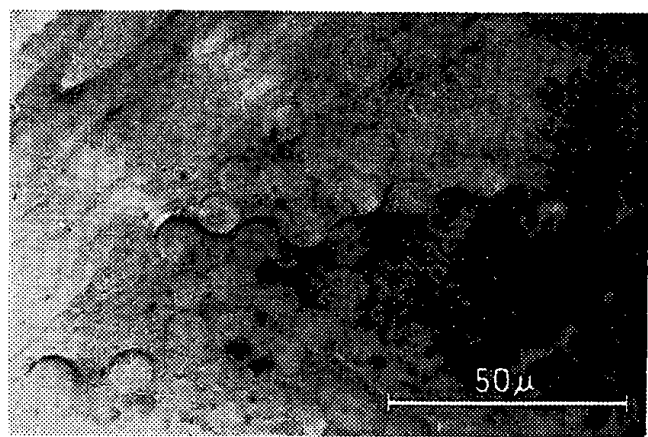

Fig. 9 Cracks inside of woof strand (enlarged) 
knee at this position are caused by the crack growth inside of the woof strands. In this figure, the curve with respect to E.I.W. is shown slightly higher than the other one. This will be due to the fact that the experiment with respect to E.I.W. was done by a somewhat rough step of strain rate. If the step were smaller, both curves would agree more closely.

Figure 11 shows the condition of the cracks inside of woof strands. The direction of the crack growth is nearly at right angles to the loading direction, and the detail about cracks is the same as Fig. 9.

4.2 Change of value of E.I.W. due to the crack growth inside of warp strands

After passing of the knee point, on account of the cracks which occurred inside of the woof strands, a constraint to the deformation of warp strand by woof strands reduces. And as the warp strand becomes free to displace, the E.I.W. $\left(W_{e} / W_{i}, W_{e} / W_{t}\right)$ begins to increase again with the increase of strain.

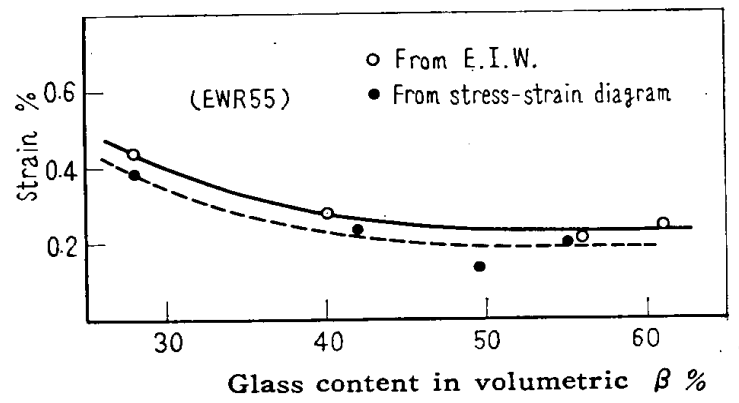

Fig. 10 Strain value at knee position

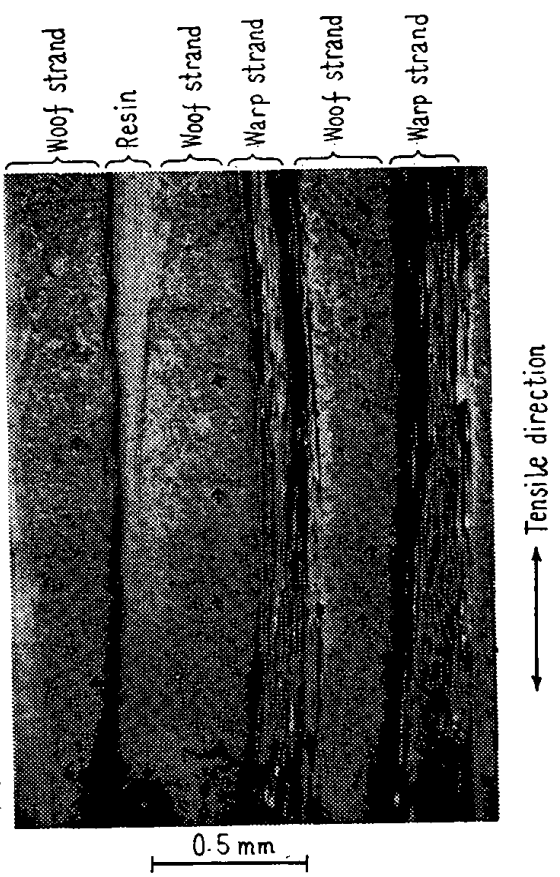

R-FRP (EWR 55), $\beta=42 \%$, stress $18 \mathrm{~kg} / \mathrm{mm}^{2}$

Fig. 11 Cracks shown with the arrows inside of woof strands
But in the case of $W_{s} / W_{t}$, the above increase falls down. a little in a certain strain range. In Figs. 5 to 7 , these strain ranges are shown by the dotted lines. The appearance of the above mentioned phenomenon almost corresponds to the instant when the cracks observable under microscope increase rapidly inside of the warp strands. In Fig. 12, an example of the total length of cracks inside of the warp strands measured by the microscope is shown on each strain value. In this figure, it becomes clear that the cracks inside of the warp strands increase rapidly for a certain value of strain.

Therefore, it may be said that the above mentioned re-falling of $W_{\mathrm{o}} / W_{t}$ is due to a rapid increase of the cracks inside of the warp strands and a reduction of the flexural rigidity against the displacement of warp strand. The relation between the strain at that instant and $\beta$ is approximately shown in Fig. 13. In the tendency of this relation, the strain value corresponding to the instant when the cracks occur becomes smaller with increase of $\beta$. But when $\beta$

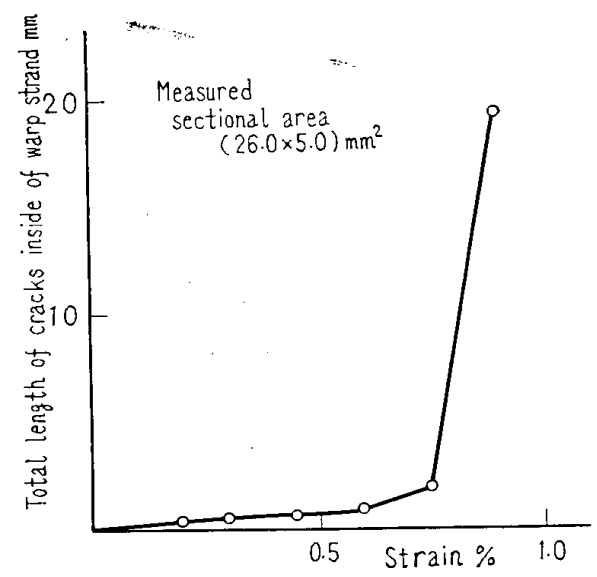

Fig. 12 Relation of increasing of cracks inside of warp strand vs. strain

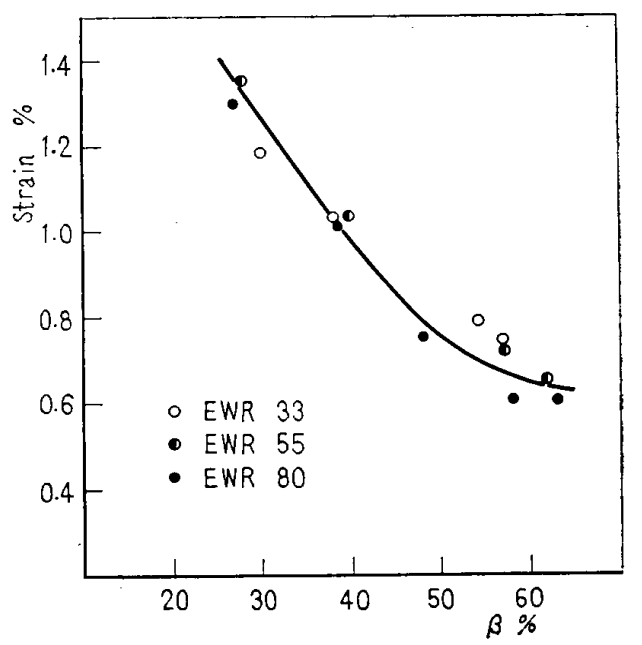

Fig. 13 Strain value for hasty increasing of cracks inside of warp strands 
becomes larger than a certain value more than about $50 \%$, the strain value when the cracks occur becomes around $0.6 \sim 0.7 \%$ irrespective of the variation of $\beta$.

However, in the case of a large value of $\beta$, it is difficult to find an exact value of the strain when the cracks occur inside of the warp strands, because of the dispersion of the results probably caused by disorders of the strands due to the pressure used in making the specimens. In Fig. 14, the state of the cracks inside of the warp strands is shown. It is similar to the state of the cracks inside of the woof strands.

When the strain exceeds the range of this changing point, $W_{e} / W_{t}$ begins to increase again. But, as the strain increases, delaminations occur partially, and on account of this, the increase of $W_{s} / W_{t}$ becomes slow and the test piece comes to a rupture soon thereafter. A phase of the delamination is shown in Fig. 15.

\subsection{Effects of the strain and $\beta$ on elastic} modulus and strain hardening

$E_{8}$ in Figs. 5 7 show the variations of elastic modulus in the form of secant modulus. The value of $E_{s}$ becomes larger with increase of $\beta$. But the variations of $E_{s}$ curves following the variation of the strain show an almost similar tendency to each other. That is to say, at first, $E_{s}$ reduces rapidly near the knee, and after that, it reduces gradually as the strain increases. And at the point about which the

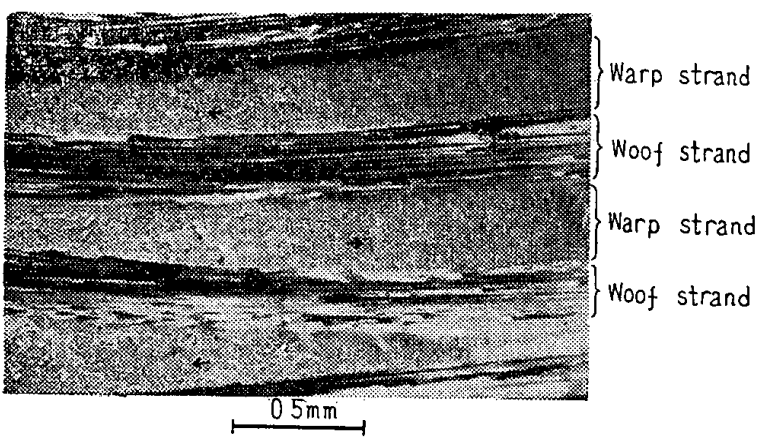

R-FRP (EWR 55), $42 \%$, stress $18 \mathrm{~kg} / \mathrm{mm}^{2}$

Fig. 14 Cracks shown with the arrows inside of warp strand

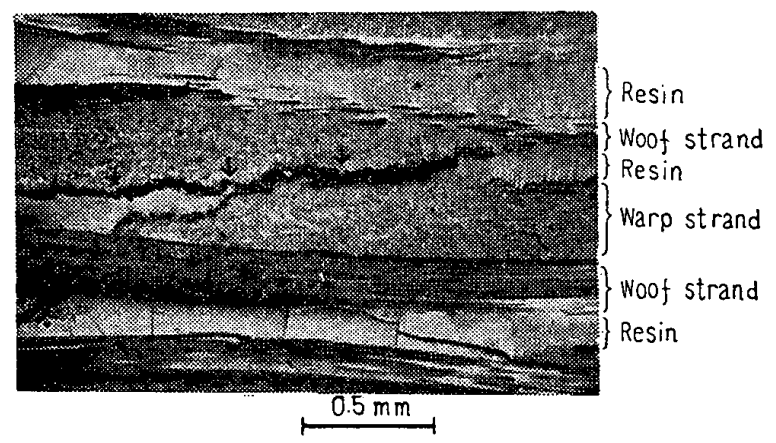

R-FRP (EWR 55), $\beta=42 \%$, stress $25.5 \mathrm{~kg} / \mathrm{mm}^{2}$ Fig. 15 Delamination at the boundary of layers cracks increase rapidly inside of the warp strands as above mentioned, it is observed that $E_{\boldsymbol{s}}$ reduces somewhat largely,

In Fig. 16, each slope of $E_{1}, E_{2}, E_{3}$ on O-L, L-K and $\mathrm{K}-\mathrm{B}$ of the stress-strain diagram shown above, is given in the arrangement with $\beta$, where, $L$ is a point at which the strain is very small $(<0.15 \%)$ and the cracks are expected not to exist yet inside of the woof strands, and $K$ is what is called the knee point at which a considerable number of cracks already exist inside of the woof strands.

According to the experimental results, the secant modulus $E_{1}$ has a rectilinear relation with $\beta$. If the secant modulus of the point where $\beta$ attains $100 \%$ on the extension of this rectilinear relation is named $E_{g}, E_{g}$ is regarded as a reduced modulus of the glass fiber considering the postures of the roving in the test piece. That is to say, it is considered that, in the range of $E_{1}$, the resin transmits the stresses between glass fibers and is subjected to a pretty high stress by stress-concentration, so it makes a behavior which seems to be affected by $\beta$ only. The proof of it is that if the straight part is extended supposing an ultimate state of $\beta=0$, it points to 0 .

$E_{2}$ and $E_{8}$ express a tendency of strain hardening. First, the value of $E_{2}$ at $\beta=100 \%$, obtained by extending the straight part of $E_{2}$, is named $\left(E_{g}\right)_{w}$. In the range of $E_{2}$, it is considered that only the warp strand of glass fiber plays a main role for elastic modulus, because the cracks already exist inside of the woof strands. So $\left(E_{g}\right)_{w}$ becomes about $1 / 2$ of $E_{g}$. The reason why $\left(E_{g}\right)_{w}$ becomes a little larger than $1 / 2$ of $E_{g}$ in practice is considered as follows.

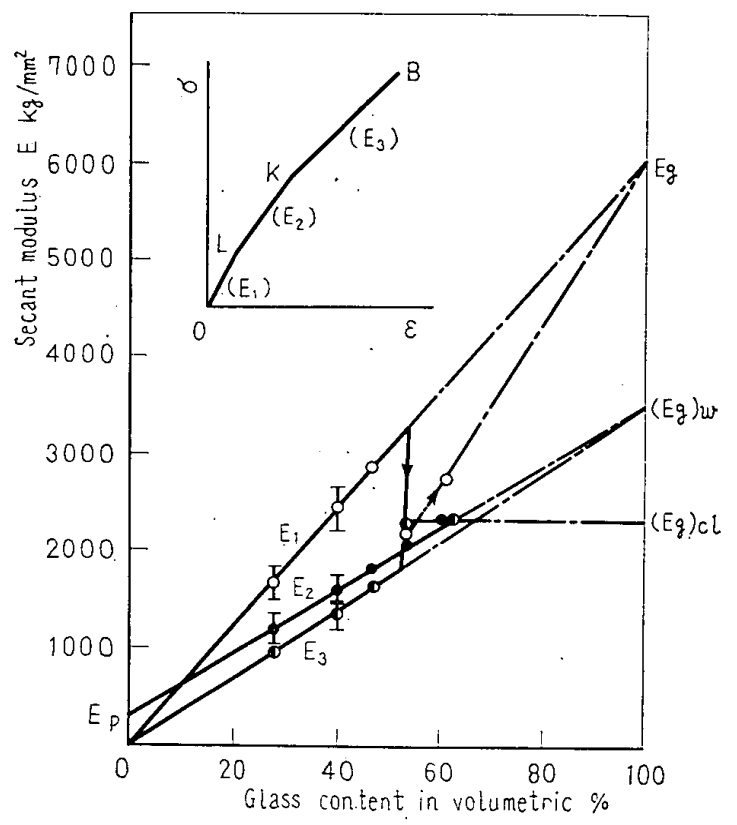

Fig. 16 Secant modulus of R-FRP 
The weight ratio of warp strands to woof strands in a fabrics is $52: 48$. And the elastic modulus of warp strand is presumed to be a little larger than that of woof strand, because the warp strand is more subjected to the tensile force than woof strand in producing fabrics. Then, the resin-layers and the warp strands are well doing in this range, so the extension of $E_{2}$ to $\beta=0 \%$ indicates the elastic modulus of the resin $E_{p}$.

In the range of $E_{3}$, equally to the case of $E_{2}$, only the warp strand plays a main role for elastic modulus, therefore the extension of the straight part of $E_{3}$ to a higher $\beta$ goes to $\left(E_{g}\right)_{w}$. But in this range, it is considered that the cracks exist already in the resin layer and warp strands, so the resin loses the action of the transmission of stresses between glass fibers, and the extension of $E_{2}$ to a lower $\beta$ goes towards 0 , unlike the case of $E_{2}$.

In this figure, transformations of $E_{1}$ and $E_{3}$ occur at about $\beta=50 \%$. For this reason, it is considered that the value of $\beta$ reaches its limitation for FRP, In the range exceeding the limitation of $\beta$, a large pressure is required in forming the specimens, and on account of that, some parts of the glass fibers are presumed to be damaged. So $E_{1}$ reduces to the value of $\beta$. The change in the glass fiber is also guessed from the fact that the tensile strength of FRP which increases first in proportion to the increase of $\beta$ comes to flatten out or reduce arround this value of $\beta$ (cf. Fig. 1).

As the reason why $E_{3}$ increases a little arround this value of $\beta$ and remains invariable after that, it is considered that the condition of laminating of the fabrics loses a normal state because of the high pressure,

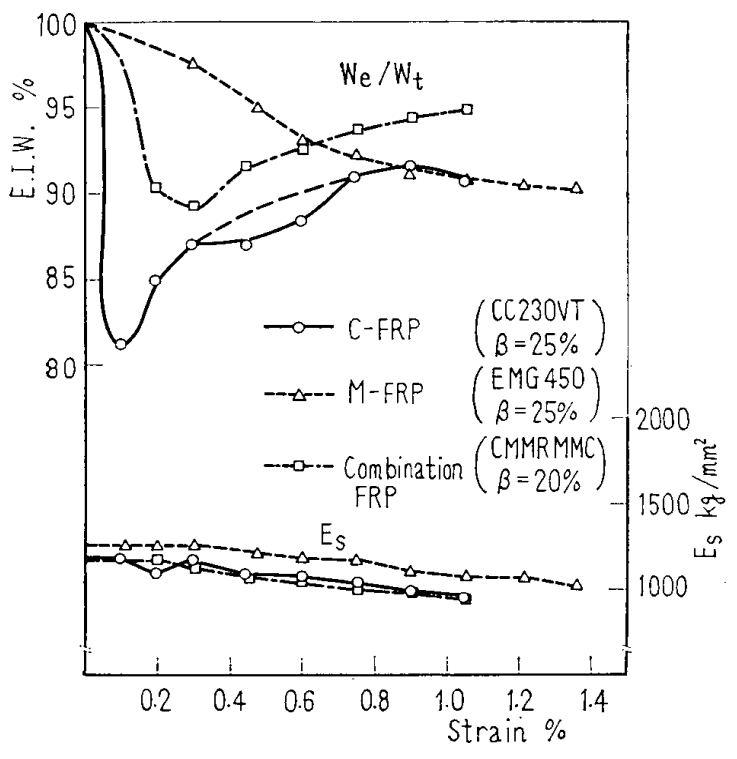

C-FRP, M-FRP, Combination FRP

Fig. 17 E.I.W. (No. 4) and neighbouring fabrics layers confuse a little and bind each other, and as the result, the stiffness of the fabrics is increased. Then, in this range of $E_{3}$, the cracks exist inside of both warp and woof strands, so the influence of the resin on elastic modulus is little. Because a layer which contains only the resin does not exist on account of the high $\beta$, it is considered that $E_{3}$ corresponding to the value of $\beta$ more than that shows the elastic modulus of glass woven fabrics $\left(E_{g}\right)_{c l}$.

\section{Elastic index of the work-done on some types of FRP}

The examined results about 3 types of FRP are shown in Fig. 17.

$5 \cdot 1$ Plastics reinforced by plain woven fabrics (C-FRP)

The tendency of the variation on the E.I.W. is nearly the same as that of R-FRP, because the reinforcements in both FRP have the same woven structure. But the knee and the changing point by the cracks inside of the warp strands occurred a little earlier than those of R-FRP. The reason for this is considered to be that the structure of plain fabrics is finer than that of the roving plain fabrics, and the conditions of stresses for woof and warp strands are different from those of the roving plain fabrics. The knee point on the stress-strain diagram shows a strain value of about $0.2 \%$ for EWR55 and about $0.1 \%$ for CC230VT (at $\beta=40 \%$ in both specimens). Wherefore, from section $4 \cdot 1$, it is considered naturally that the strain value where the large decrease of the E.I.W. appears for C-FRP is lower than that of RFRP.

In the same consideration, the strain value at the changing point with increase of the cracks inside of the warp strands is lower than that in the case of R-FRP.

$5 \cdot 2$ Plastics reinforced by non-woven fabrics (M-FRP)

Non-woven fabrics is a fabrics which consists of a disordered distribution of chopped strands and is quite different from the plain fabrics. The experimental results of the E.I.W. have large differences from those of R-FRP, and they behaved as semihomogeneous materials. The E.I.W. decreases a little rapidly from the strain value about $0.3 \%$ and continues to decrease as the strain increases. Then the E.I.W. does not show any recovery as in the case of R-FRP.

5.3 Plastics reinforced by combinations of some types of glass fabrics (Combination FRP)

As above mentioned, there is a large difference between the values of E.I.W. of R-FRP and M-FRP. 
In practical cases, the plastics reinforced by both roving plain fabrics and non-woven fabrics are used for many purposes. It is expected naturally that the behavior of E.I.W. of such a combined FRP is affected by the quantities of both fabrics. The result on the FRP constituted by CMMRMMC is shown in Fig. 17. The decreases of E.I.W. at the knee is little by the influence of the quantity of $M$. In a range of large strain, the curve of E.I.W. becomes slightly higher by the influences of $R$ and $C$.

\section{Several factors which affect the elastic index of the work-done}

\subsection{The influence of the volumetric ratio} of glass fiber

The influences of $\beta$ on E.I.W. of R-FRP (3 types) are shown in Fig. 18. The values of E.I.W. at the knee point are nearly constant for various values of $\beta$ of specimen up to about $40 \%$ considered as the range of handlay-up method. We consider that this constant value of E.I.W. will be obtained in the condition under which the influence of low $\beta$ and the decrease of cracks cancel each other, because the layer of resin only exists in the FRP in this range of $\beta$, and this layer is not related with the cracks inside of the woof strands.

As $\beta$ increases more than $50 \%$, the layer of resin only vanishes gradually, and the decrease of E.I.W. at the knee point becomes little. For example, the behavior of E.I.W. in the specimen which consists of EWR55 and has $61 \% \beta$ is very elastic in spite of

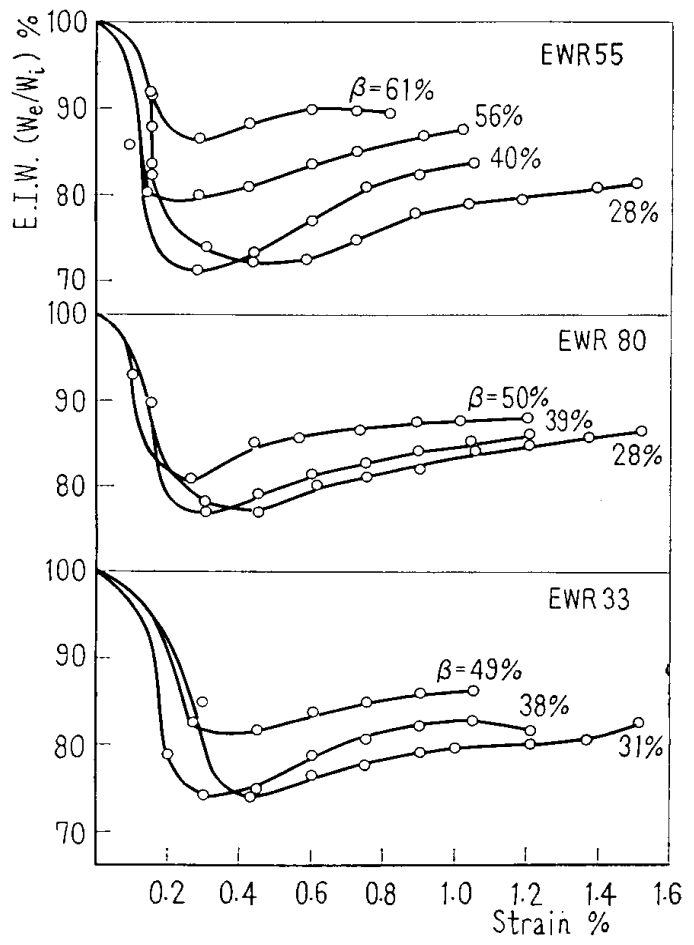

Fig. 18 Influence of $\beta$ on E.I.W. the fact that many failured glass fibers caused by forming pressure are expected to exist in this specimen judzing from its tensile strength (Fig. 1).

When the strain passes the knee and reaches a large value, E.I.W. increases with an increase of $\beta$ :

\subsection{Influence of strain speed}

Figure 19 shows the influence of the strain rate in the range $0.20 \sim 1.25 \mathrm{~min}^{-1}$ on $\mathrm{R}-\mathrm{FRP}$ (EWR55) and M-FRP. It is considered from this figure that the variation of the strain rate does not affect $R-F R P$, and affects slightly M-FRP.

On the factors of the influence of strain rate, it is conjectured that the effect of the stress concentration becomes larger with a higher strain rate in the matrix of resin, then cracks grow quickly in the resin. But from this examination, we can not draw any clear conclusion on the influence of strain rate, since the strain rate is not used within a wide range.

6.3 Influence of the kinds of reinforcement

According to those mentioned in Chapters 4 and 5 , in the plastics reinforced by fabrics which has some woven structure as plain fabrics, each E.I.W. is slightly different individually by the kind of fabrics. But it shows the same tendency from a general view point. On the other hand, E.I.W. of M-FRP shows a large difference from above FRP in the effect of strain especially a small strain.

\section{Conclusions}

We studied the elasto-plastic behaviors of FRP using their E.I.W. The results obtained are as follows:

(1) When the plastics is reinforced by the

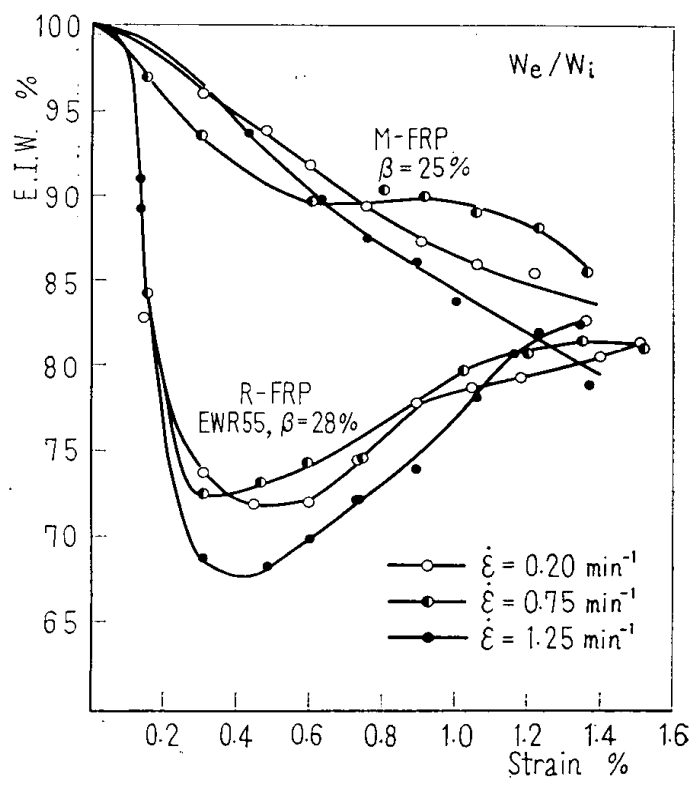

Fig. 19 Influence of strain speed on E.I.W. 
glass fabrics which has a systematic woven structure, E.I.W. at the knee point decreases remarkably under tensile loading. This decreasing is due to the growth of cracks inside of the woof strands.

(2) The strain passes through the knee, the value of E.I.W. recovers again, even though it has a region in which E.I.W. shows a little decreasing temporarily. This decreasing position corresponds to the instant when the cracks inside of the warp strands increase rapidly, and the decreasing of E.I.W. is caused by these cracks.

(3) The value of E.I.W. becomes larger for a larger value of $\beta$. But the amount of decreasing of E.I.W. at the knee shows a constant value approximately for $\beta$ up to $40 \%$.

(4) The value of E.I.W. of M-FRP decreases with the increase of strain and shows the same behavior as that of semi-homogenous materials.

(5) In the Combined FRP, the value of E.I.W. shows some different behaviors affected by the kind and quantity of the reinforcement.

In the above mentioned results, the behaviors and the failure mechanism for the strained plastics reinforced by some kinds of glass fabrics have been clarified mostly. From this, it will be easy to select the kinds or combination of reinforcements for practical use of FRP. R-FRP has a large tensile strength, and is promising as a shock-absorbing material for its inelastic behavior in a small strain region. On the other hand, non-woven fabrics will make important reinforcement to compromize the strength with the cost of FRP.

\section{References}

(1) Besal, M.B. and Mcgarry, F.J., ASTM Bull., TP $156(1956)$.

(2) Fujii, T. and Masuda, Y., The 5th symposium on lightened structures (in Japanese), (1964), p. 187.

(3) Tsai, S.W. and Azzi, V.D., AIAA Jour., Vol. 4, No. $2(1966-2)$, p. 296.

(4) Hirai, T., et al., Collected papers of annual meeting of Japan Texile Machinery Soc.(in Japanese), (1961), p. 97.

(5) Fujiwara, S., et al., Trans. Industrial Research Institute Osaka Pref. (in Japanese), No. 46 (1968$11)$, p. 33

(6) Otsuki, S., Preprint of Japan Soc. Mech. Engrs. (Kansai) (in Japanese), (1958-3), p. 24.

(7) Otsuki, S., et al., Preprint of Japan Soc. Mech. Engrs. (Kansai) (in Japanese), 2nd room (1967-3), p, 37.

(8) Yoshida, H., Reference (7), p. 40.

(9) Otsuki ${ }_{2}$ S., et al., Reference (7), p. 30.

(10) Fujiwara, S., et al., Preprint of Japan Soc. Mech. Engrs. (in Japanese). No. 187. (1968-4), p. 133.

(11) Fujino, K., et al., Trans. Japan Textile Mechinery Soc. (in Japanese), Vol. 15, No. $2(1962-2)$, p. 100; Vol. 15, No. 3 (1962-3), p. 202.

(12) Fujiwara, S., et al., Preprint of Japan Soc. Mech. Engrs. (Kansai No. 223), (in Japanese), 1st collection $(1969-11)$, p. 4 